\title{
Prozessintensivierung
}

\section{Mehr machen mit weniger - Intensivierung von Ganzzell-Bioprozessen}

\author{
FRANK HOLLMANN ${ }^{1}$, ROLAND ULBER ${ }^{2}$, DIRK HOLTMANN ${ }^{3}$ \\ 1 DEPARTMENT OF BIOTECHNOLOGY, DELFT UNIVERSITY OF TECHNOLOGY, DELFT, \\ NIEDERLANDE \\ 2 LEHRGEBIET BIOVERFAHRENSTECHNIK, TU KAISERSLAUTERN \\ 3 INSTITUT FÜR BIOVERFAHRENSTECHNIK UND PHARMAZEUTISCHE TECHNOLOGIE, \\ ARBEITSGRUPPE INTENSIVIERUNG VON BIOPROZESSEN, TECHNISCHE HOCHSCHULE \\ MITTELHESSEN, GIESSEN
}

In chemical process engineering, process intensification (PI) has proven itself as a method that resulted very often in processes with an at least doubled process performance. In recent years, the PI techniques have found more and more applications in biotechnology. Exemplary continuous processes, single-use reactors, electrobiotechnology and hybrid techniques are discussed here.

DOI: $10.1007 / \mathrm{s} 12268-021-1508-6$

(c) Die Autoren 2021

In den letzten Jahren hat der Einsatz der Prozessintensivierung (PI) in der chemischen Verfahrenstechnik zu einer Vielzahl von innovativen Verfahren geführt. Allgemein ist PI ist auf material-, zeit- und energieeffizientere Technologien ausgerichtet. Weiterhin zielt PI auf sicherere und nachhaltige technologische Entwicklungen ab. In der European Roadmap of Process Intensification [1] wurde 2007 eine allgemeine Definition der PI aufgezeigt „Prozessintensivierung ist die Zusammenstellung von oft radikal innovativen Prinzipien (Paradigmenwechsel) im Prozess- und Ausrüstungsdesign, die bedeutende Vorteile in Bezug auf Prozesseffizienz, Kapital- und Betriebskosten, Qualität, Abfallmengen und Prozesssicherheit mit sich bringen“. Im Gegensatz zur klassischen Prozessoptimierung werden in der PI meist Verbesserungen der Performanz um mindestens den Faktor zwei angestrebt. Typische Ansätze in der PI sind die Reduzierung der Anzahl der Apparaturen (Integration mehrerer Funktionalitäten), die Verbesserung des Wärmeund Massentransfers durch fortschrittliche Mischtechniken und kürzere Diffusionswege, Miniaturisierung, neue Verfahren des Energieeintrags, neue und integrierte Trennverfahren sowie eingebettete Optimierungsund Kontrollstrategien. In den letzen Jahren kommen auch in der Bioverfahrenstechnik verstärkt die Methoden der Prozessintensivierung zum Einsatz. Dabei werden sowohl Prozessen mit isolierten Enzymen als auch Ganzzellkatalysatoren betrachtet. In dieser Kurzübersicht werden entsprechende Verfahren mit Ganzellkatalysatoren vorgestellt, in der nächsten Ausgabe (BIOspektrum 2/21) werden intensivierte Verfahren mit isolierten Enzymen erläutert. Die Abbildung 1 zeigt eine schematische Übersicht über die Anwendung der PI in Prozessen mit Ganzzellbiokatalysatoren. Die PI in der chemischen Verfahrenstechnik und der Bioverfahrenstechnik weisen teilweise Überlappungen auf (z. B. Membran-Adsorber, Rotating-BedReaktoren). Es gibt aber auch große Unterschiede, wie z. B. der Einsatz von Single-useReaktoren oder beim Einsatz von alternativen Lösungsmitteln. Im Folgenden werden drei Beispiel erläutert.

\section{Biopharmazeutische Produktion -} kontinuierliche Prozesse und Singleuse-Reaktoren

Bei den Herstellungsverfahren in der biopharmazeutischen Industrie werden bisher meist Unit-Operationen im Batch-Modus kombiniert. Parallele Ziele im Sinne der PI sind hier die Verbesserung der Produktqua- lität, die Reduktion sowohl des Prozessmaßstabs als auch der Anzahl der Unit-Operations. Final wird damit eine Kostensenkung der gesamten Produktion angestrebt. In den letzten Jahren haben sich eine Reihe von Optionen für die Intensivierung von Bioprozessen in der biopharmazeutischen Industrie herausgebildet. Dazu gehören intensivierte Fed-Batch-Prozesse, kontinuierliche Perfusion und Zellkulturen mit intensivierter kontinuierlicher Perfusion. Viele biopharmazeutische Unternehmen erachten gerade kontinuierliche Perfusionsverfahren in EinwegBioreaktoren (single-use) als geeignete Strategie zur Erreichung der Ziele. Die Prozesse in kleineren Einweg-Bioreaktoren sollen dann anstelle von etablierten Prozessen in größeren Edelstahlgefäßen durchgeführt werden [2]. Laut eines Kostenanalysemodells der Fa. Roche kann der Einsatz von Medienkonzentraten und optimierten Medien zu einer vierfachen Erhöhung des Titers bei kürzeren Prozessdurchläufen führen, was die Reduzierung eines 12.000-Liter-Prozesses auf 2.000 Liter ermöglicht, welcher dann in Einweg-Bioreaktoren durchgeführt werden kann [2]. In den letzten Jahren wurden dabei die Einweg-Bioreaktoren kontinuierlich verbessert, z. B. durch das Re-Design von Rührwerken, die einen verbesserten Stoffaustausch ermöglichen. Der Einsatz von EinwegBioreaktoren kann zu geringeren Kapitalund Betriebskosten, einem kleineren Platzbedarf und größerer Flexibilität führen. Besonders hervorzuheben sind hier die die beschleunigte und einfachere Vorbereitung der Reaktionssysteme sowie die kürzeren Prozesszeiten. Dieses führt zu einem verringerten Einsatz an Personal und Ressourcen - eben zu mehr Produkt bei verringertem Aufwand.

Elektrobiotechnologie - Elektrifizierung der Bioproduktion zur Nutzung von $\mathrm{CO}_{2}$ als Kohlenstoffquelle

Der steigende Anteil der erneuerbaren Energien führt zu einem zunehmenden Speicherbedarf überschüssiger elektrische Energie. Neben der Batterietechnologie als Möglich- 
keit der Speicherung bietet sich auch die elektrochemische Synthese als Technologie zur direkten Nutzung an (Power-to-X). Bei der Nutzung von $\mathrm{CO}_{2}$ als Rohstoff für die elektrochemische Synthese ist das Spektrum an möglichen Produkten jedoch begrenzt. Um eine wertschöpfende Synthese ausgehend von $\mathrm{CO}_{2} \mathrm{zu}$ ermöglichen, bieten sich elektrobiotechnologische Synthesen an. Solche Verfahren bieten als besondere Vorteile die Nutzung von klimaschädlichen Abgasströmen als Rohstoff für die Herstellung höherwertiger Produkte unter milden Bedingungen mit hoher Selektivität. Kürzlich wurde ein Verfahren zur elektrochemischen Reduktion von $\mathrm{CO}_{2}$ zu $\mathrm{CO}$ und eine nachgeschaltete mikrobielle Umsetzung mit Clostridien z. B. zu Butanol und Hexanol vorgestellt [3]. Diese Produkte werden z. B. für die Herstellung von Spezialkunststoffen oder Nahrungsergänzungsmitteln benötigt. Der Laborprozess wird aktuell von den Unternehmen Evonik und Siemens in eine technische Versuchsanlage mit einem $\mathrm{CO}_{2}$-Elektrolyseur mit einer Gesamtelektrodenfläche von $3.000 \mathrm{~cm}^{2}$ und einem 2-m ${ }^{3}$-Bioreaktor skaliert. Verschiedene Autoren haben gezeigt, dass mit weiteren Produktionsorganismen auch komplexere Moleküle in elektrobiotechnologischen Prozessen produziert werden können. Das Bakterium C. necator ist in der Lage, Kohlenstoffdioxid als C-Quelle zu nutzen. Die benötigten terminalen Elektronenakzeptoren $\left(\mathrm{O}_{2}\right)$ und die Energiequelle $\left(\mathrm{H}_{2}\right)$ lassen sich durch Wasserelektrolyse erzeugen. Dies wurde zum Beispiel zur elektrobiotechnologischen Produktion von Terpenen genutzt [4]. Weiterhin wird Formiat als ein ideales Intermediat für eine elektrochemische Umsetzung von $\mathrm{CO}_{2}$ und nachfolgende biotechnologische Veredelung angesehen [5], dies wurde z. B. zur Produktion der PolymerVorstufe Mesaconsäure mit M. extorquens [6] oder Synthese des Bioplastiks PHB mit C. necator [7] genutzt. Die Verbindung von Elektrochemie und Biotechnologie zur Nutzung von Strom und $\mathrm{CO}_{2}$ stellt im Vergleich zu etablierten Verfahren einen Paradigmenwechsel dar und führt zu effizienten Verfahren, welche knapper werdende Ressourcen optimal nutzen.

\section{Hybride Bioprozesse - Kombination von Reaktion und Aufreinigung}

Der Einsatz von hybriden bzw. integrierten Unit-Operationen kann häufig zu einer Intensivierung von Prozessen führen; bei biotechnologischen Prozessen stehen dabei insbe-

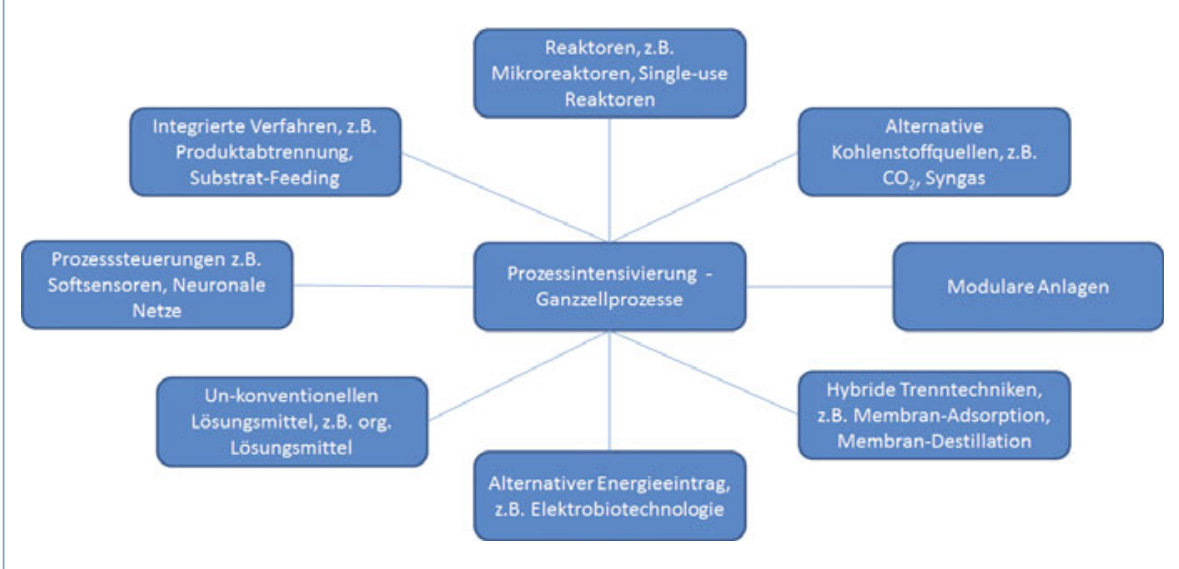

Abb. 1: Beispiele für Methoden und Werkzeuge der Prozessintensivierung in der Bioverfahrenstechnik bei der Anwendung von Ganzzellprozessen.

sondere die Integration von mikrobieller Produktion und Trennprozessen im Fokus. Ziel dieser in situ-Produktabtrennung (ISPR, in situ-product removal) ist die schnelle Abtrennung der Reaktionsprodukte von den Produktionsorganismen zur Vermeidung negativer Einflüsse auf die Zellen. Beispielsweise wird die Produktivität an Biobutanol durch die Toxizität des Produkts auf Produktionsorganismen limitiert. Die in situ-Produktabtrennung von Butanol kann dann die Produktivität verbessern. Bei entsprechenden Aceton-, Butanol- und Ethanol-Fermentationen (ABE-Fermentationen) werden daher Gas-Strippig, Vakuumfermentation, Pervaporation, Flüssig-Flüssig-Extraktion, Perstraktion und Adsorption eingesetzt [8]. Diese Verfahren wurden beispielsweise auch zur Intensivierung der Bioproduktion von Itaconsäure [9], Milchsäure [10] und 2-Phenyelthanol [11] genutzt. Der Einsatz der ISPR-Technik hat jeweils zu Verbesserungen der Ausbeute und/oder Produktivität geführt - wiederum wurde das Ziel, mehr Produkt bei geringerem Rohstoffeinsatz zu produzieren, durch die PI erreicht.

\section{Zusammenfassung}

Während die PI in der chemischen Technik schon seit langem etabliert ist, setzen sich die Konzepte zunehmend auch in der Bioprozesstechnik durch. Dies wird sowohl vor dem Hintergrund eines erhöhten Kostendrucks als auch der Schonung der Ressourcen zunehmend wichtiger. PI benötigt oft die multidisziplinäre Zusammenarbeit, um in „kreativen“ Prozessen immer wieder einen Paradigmenwechsel voranzutreiben.

\section{Literatur}

[1] ERPI (2007) European roadmap for process intensificatIon. Download: https://efce.info/efce_media/-p-531.pdf

[2] Eisenkratzer D (2019) Cell retention and harvest technologies for efficient high-cell-density processing. BioProcess Int 7: 13-14
[3] Haas T, Krause R, Weber R et al. (2018) Technical photosynthesis involving $\mathrm{CO} 2$ electrolysis and fermentation. Nat Catal 1: 32-39

[4] Krieg T, Sydow A, Faust S et al. (2018) CO2 to terpenes: autotrophic and electroautotrophic $\alpha$-humulene production with cupriavidus necator. Angew Chem Int Ed 57: 1879-1882 [5] Yishai O, Lindner SN, Gonzalez de la Cruz J et al. (2016) The formate bio-economy. Curr Opin Chem Biol 35: 1-9 [6] Hegner R, Neubert K, Kroner C et al. (2020) Coupled electrochemical and microbial catalysis for the production of polymer bricks. ChemSusChem 13: 5295-5300

[7] Stöckl M, Harms S, Dinges I et al. (2020) From CO(2) to bioplastic - coupling the electrochemical $\mathrm{CO}(2)$ reduction with a microbial product generation by drop-in electrolysis. ChemSusChem 13: 4086-4093

[8] Outram V, Lalander CA, Lee JGM et al. (2017) Applied in situ product recovery in ABE fermentation. Biotechnol Prog 33: 563-579

[9] Kaur G, Maesen M, Garcia-Gonzalez L et al. (2018) Novel intensified back extraction process for itaconic acid: toward in situ product recovery for itaconic acid fermentation. ACS Sustainable Chem Eng 6: 7403-7411

[10 Fan R, Ebrahimi M, Czermak P (2017) Anaerobic membrane bioreactor for continuous lactic acid fermentation. Membranes 7: 26

[11] Červeňanský I, Mihal' M, Markoš J (2020) Pertractionadsorption in situ product removal system: intensification of 2-phenyelthanol bioproduction. Separation and Purification Technology 251: 117283

Funding note: Open Access funding enabled and organized by Projekt DEAL Open Access: Dieser Artikel wird unter der Creative Commons Namensnennu 4.0 International Lizenz veröffentlicht, welche die Nutzung, Vervielfältigung, erlaubt, sofern Sie den/die ursprünglichen Autor(en) und die Ouelle ordnungsgem ordnungsgemäß̈ nennen, einen Link zur Creative Commons Lizenz beifügen
angeben, ob Änderungen vorgenommen wurden. Die in diesem Artikel enthaltenen Bilder und sonstiges Drittmaterial unterliegen ebenfalls der genannten Creative Commons Lizenz, sofern sich aus der Abbildungslegend nichts anderes ergibt. Sofern das betreffende Material nicht unter der genannten Creative Commons Lizenz steht und die betreffende Handlung nicht nach gesetzlichen Vorschriften erlaubt ist, ist für die oben aufgeführten Weiterverwendungen des Materials die Einwilligung des jeweiligen Rechteinhabers einzuholen. Weitere Details zur Lizenz entnehmen Sie bitte der Lizenzinformation auf http://creativecommons.org/licenses/by/4.0/deed.de.
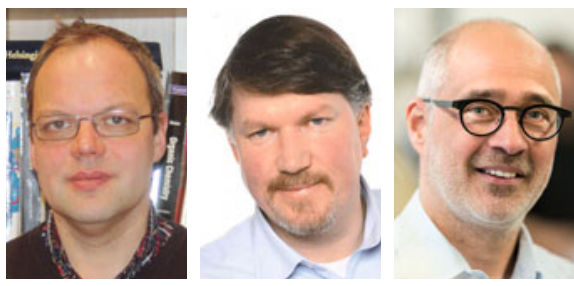

Frank Hollmann, Roland Ulber und Dirk Holtmann (v.l.n.r.)

Korrespondenzadresse:

Prof. Dr.-Ing. Dirk Holtmann

Fachbereich LSE

Institut für Bioverfahrenstechnik und Pharmazeutische Technologie

Technische Hochschule Mittelhessen

Wiesenstraße 14

D-35390 Gießen

dirk.holtmann@lse.thm.de 